\title{
ADVANCES IN THE PHYSIOLOGY OF PERIPHERAL NERVE
}

\author{
By D. K. Hill \\ Lately physiologist at the Plymouth Laboratory
}

The last 20 years have seen many advances in our knowledge of the mechanisms responsible for the transmission of impulses in peripheral nerve fibres. This has come about largely through researches on the nerves of marine invertebrates. Much of the work has been done at Plymouth. The purpose of this article is to give some of the reasons why certain features of these nerves, which distinguish them from the nerves of vertebrates, should make them so desirable for experimental work.

The giant nerve fibre of the squid did not come into the limelight until the middle nineteen-thirties, nor did the other smaller single fibres which can be dissected out of the nerve trunks of cuttlefish, crabs and lobsters. Before describing the work with single fibres, we will go further back to about 1926 when, as the result of work by A. Levin at Plymouth, it was realized that the multi-fibred limb nerves of Crustacea were highly suitable for studies of the effect of activity on nerve metabolism.

\section{MUlTi-FIBREd INVERTEBRATE Nerves}

For many years it had been believed that in the transmission of nerve impulses no heat is evolved. Until the end of the last century practically all views attributed to nerve a completely passive role in the conduction of an impulse. As recently as 25 years ago the impulse was pictured, in Sir William Bayliss's words, as a 'reversible physico-chemical process, not associated with loss of material on account of metabolic reactions'. However, this was wrong. Heat is set free, but its amount was too small to detect. A. V. Hill finally demonstrated this phenomenon in frog's nerve: but, as he said (in I932): 'physiological laboratories ought to be built near the sea, or at least within reach of aquaria where delicate marine animals could be stored. The heat production of crab's nerve could have been measured 20 years ago: actually I4 years were wasted before it was measured in frog's nerve.' Whereas, by 1930, it had become possible to measure the heat production following a single impulse in the crab's nerve, in frog's nerve, where much less is produced, roo impulses are required.

Heat production is only one sign of metabolism. In every aspect the extra metabolism due to stimulation of a crustacean nerve is much greater than it is in the frog's nerve. This superior efficiency of the frog's nerve is evident in 
its comparative resistance to fatigue: it can continue to pass impulses at a high frequency for a long time, while the crab's nerve fatigues rapidly. We now understand the reason for this difference. The fibre of a vertebrate nerve possesses a myelin sheath, composed of lipoid material, which in effect 'insulates' the fibre except at the nodes of Ranvier, where the sheath is absent. It has recently been shown that the nervous impulse progresses by 'jumping' from node to node. This means that the active process, which spreads over the whole membrane in the invertebrate non-myelinated fibre, affects only that part of the frog fibre which is free from the myelin covering, so it is only in the region of the nodes that any extra metabolism occurs when the fibre becomes active. The whole of the inter-nodal part of the fibre is a purely passive bridge with quite simple electrical properties, doing no more than enable one node to excite the next. The frog fibre is only Io $\mu$ across, but the nodes may be $2 \mathrm{~mm}$. apart, so the area of membrane involved in activity is very small. Apart from the greater economy which goes with this ingenious mechanism, there is the added advantage that considerably faster transmission is possible with a given size of fibre.

The two features of invertebrate nerve which the physiologist finds so much to his advantage, namely the high rate of metabolism and the occasional appearance of large fibres, might neither have been found if the myelin sheath had been developed in this type of nerve. One can think of only two possible reasons why the large fibre evolved: first, because a large fibre conducts faster than a small one, and at a higher frequency; secondly, because a large fibre is more resistant to fatigue than a small one, for the surface activity is supported by a relatively larger volume of axoplasm (the name given to the interior substance of a nerve fibre). One advantage of fast transmission is plain enough, reaction times are reduced; but there is another, more subtle, reason which may be suggested. A large muscle mass as, for instance, the mantle muscle of the jet-propelled squid, may have to be synchronously activated in all its parts from one ganglion. Low-velocity fibres would produce a wave of contraction, inefficient for propulsion, while high-velocity fibres can be sufficiently graded in size (the larger, faster fibres going to the more distant part of the muscle) to provide a synchronous burst of impulses at all nerve endings in the muscle.

Turning to the question of resistance to fatigue, we now know enough about the actual quantities of ions traversing the active membrane to be able to calculate the extent to which a nerve fibre is 'run down' when an impulse passes along it. The fibre loses some of its internal potassium and gains sodium from the outside when it is stimulated, and it takes many minutes for the original balance to be restored. The nerve can pass roo impulses per sec. (the giant fibres up to 500 per sec.), but the axoplasm of the smallest fibres does not constitute a sufficient reserve for prolonged activity at high frequency; even after $10-20 \mathrm{sec}$. perhaps as much as a half of the internal potassium would 
be lost from a I $\mu$ fibre. However, the very small fibres probably have a sensory function, or at any rate a function which normally requires them to pass but few impulses in a minute, so the axoplasm may well provide an adequate reserve. On the other hand, the muscles of the leg require innervation by nerve fibres which are less prone to fatigue, for they must be capable of passing impulses at high frequency, and bursts of activity may be required in quick succession.

It is not possible to be sure which of these factors has been predominant in the evolution of the larger motor fibres. Both the resistance to fatigue and the higher velocity of transmission could have been achieved by the myelin sheath, but luckily for the physiologist, another and more clumsy solution was found for the invertebrate!

Within the last I5 years advances have been made in our knowledge of the ionic equilibrium which determines the electrical potential across the membrane, and in connexion with the passage of ions across this membrane when the nerve is stimulated. It has been possible, for instance, to measure the amount of potassium which escapes from the fibres of a crustacean nerve when it is stimulated. It is improbable that this could have been done with a frog's nerve, for the ionic currents, being presumably confined to the small areas of membrane around the nodes of Ranvier, would be too small to measure.

There is another sign of activity, easily measured in a non-myelinated nerve trunk, which is so small as to be quite undetectable in a frog's nerve: this is the change in the intensity of light scattered by the nerve when it is stimulated; it is thought to be caused by a minute swelling of the individual nerve fibres.

\section{ISOLATED NeRVE Fibres}

Research with single fibres started about I935, following J. Z. Young's histological studies of the nerves of Crustacea and Cephalopods. Before describing some of the things which can be done with the giant fibre of the squid, reference will be made to the work with isolated crustacean nerve fibres. The great majority of the fibres of a crustacean nerve trunk, tens of thousands of them, are very small, having diameters between 0.5 and $5 \mu$; the function of these fibres is not certain, but they are probably mostly connected with sensory endings. In addition to the very small fibres there are larger ones supplying the muscles. These motor fibres are relatively very few, being numbered in tens rather than in thousands, and the largest are $25-80 \mu$ in diameter. By skilful dissection these large fibres can be isolated singly, they can be manipulated without much difficulty, and will survive and respond to stimulation for many hours in artificial saline solution. A fibre of this sort is not large enough for insertion of a micro-electrode, nor is it suitable for the other purposes which make the squid fibre so useful, such as the chemical analysis of the constituents of the axoplasm. In other words, the single 
crustacean fibres can only be operated on by techniques generally used for multi-fibred bundles of nerves. What, then, is gained by using the single fibre? Why would not bundles of smaller fibres-much more easily prepared-serve just as well? Briefly, the answer can be given as follows. The physiology of peripheral nerves has reached a stage where really accurate quantitative facts and figures are required. These cannot be obtained from the multi-fibred preparation, as some examples will show. (i) Inorganic ions cross the membrane of a nerve fibre when it is stimulated: we want to know, accurately, the quantities of ions which pass a unit area of membrane per impulse. These can be measured by radio-active tracers and other methods. The difficulties of doing this accurately with a bundle of fibres are obvious enough. The fibres are of different sizes, one cannot be certain that they are all excitable, and complicated (and approximate) corrections have to be made for diffusion between the inter-fibre spaces and the exterior bulk of the solution. (ii) The electrical resistance and capacity of the nerve membrane must be measured. This can be done accurately only with a single fibre, where all the quantities involved can be determined precisely. (iii) We wish to be able to make a rapid change in the composition of the fluid surrounding the fibre. With fibres in a bundle this is not possible, for diffusion is too slow; the ions or other chemical substances would not enter or leave the inter-fibre spaces sufficiently rapidly. (iv) For certain purposes it is necessary to work with a fibre which is surrounded by only a very small volume of electrolyte solution: to do this the fibre is transferred into paraffin oil. Under such conditions it still conducts, even though the external solution occupies a layer only a few microns thick.

\section{The Giant Fibre}

The mantle muscles of the squid and cuttlefish are supplied by nerve fibres which radiate out from the stellate ganglia. These are the so-called giant fibres. The largest are found in the squid: they may be $500-800 \mu$ in diameter. By virtue of their large size they allow certain procedures which are not applicable to smaller fibres. An isolated fibre can be cannulated at its cut end, and a thin rod-like electrode (or even a double electrode, consisting of two independent wires wound helically around a fine glass rod) can be inserted through the cannula, and pushed down so far into the interior of the fibre that the active part of the electrode may be some centimetres from the cut end. Surprisingly enough, this operation does not destroy the excitability of the fibre, and it may conduct impulses, and to all intents and purposes be in perfect condition, for many hours after the electrode has been inserted.

The reason for wanting an internal electrode can be explained as follows. In the unstimulated fibre there is a potential difference across the membrane amounting to about $50 \mathrm{mV}$. This is known as the 'resting potential' and is in the sense of the interior of the fibre being negative with respect to the outside. 
In a multi-fibred nerve trunk, or in a small fibre, the resting potential cannot be measured accurately, because it is difficult to make electrical connexion with the axoplasm in the intact part of the fibre which is conducting the impulse. The best that can be done is to make connexion with the axoplasm at the ends of the fibre: this is achieved by some form of chemical or mechanical injury to the ends, which has the effect of breaking down the electrical resistance of the membrane, thus allowing more or less direct connexion with the inside. But, owing to short circuits, one can only tap off a rather unknown fraction of the true membrane potential in this way. The insertion of an electrode into the inside of a giant fibre is a means of overcoming the difficulty; one can then record the full potential, either at rest or during the passage of an impulse. Leading off from the outside of the fibre is easily achieved; all that is needed is an electrode in the conducting electrolyte solution in which the active part of the fibre is bathed. An amplifier and cathode-ray tube are used for recording the membrane potential.

The earliest experiments with the internal electrode led to a rather surprising discovery. It had always been tacitly assumed that activity simply involved a transitory disappearance of the resting potential. Recordings made with external electrodes were not sufficiently reliable for this assumption to be seriously challenged. Experiments with the internal electrode showed that the membrane potential actually reverses its sign as the impulse passes. Further experiments have suggested that the reversal is caused by a large increase in the permeability of the membrane to sodium ions. The concentration of sodium ions in the axoplasm is very low, so there is a gradient tending to drive them in from the outside, and the negatively charged interior of the resting fibre also tends to draw in the positively charged sodium ions. A sudden breakdown in the barrier will allow sodium ions to rush in, the membrane potential will fall as the negative charge inside is satisfied by the positive sodium ions, and if the permeability becomes high enough, and the increase is preferentially in favour of sodium, the potential will actually reverse-but one gets into rather deep water here in attempting to explain it. At all events, there is now good support for the view that the action potential is primarily due to a sudden inrush of sodium. To elucidate this matter further, A. L. Hodgkin, A. F. Huxley and B. Katz have used a double internal electrode, one wire of which forces the membrane potential to change in a prescribed manner-it constrains the membrane, as it were, to pass through an abnormal action potential cycle-and the other wire records the current which flows across the membrane as the result of this. The problem, both from the technical and theoretical aspects, has become rather complicated; the findings have not yet been published.

Another facility is afforded by the large size of the squid fibre. The volume of the axoplasm is sufficient to allow analysis of its constituents. The quantities of the various inorganic ions can be estimated, the identities and amounts of the internal amino-acids, which play a part in maintaining the ionic balance, 
can also be determined. The axoplasm is squeezed out of the fibre for such analyses. It is found to be of a jelly-like consistency, and other than straight chemical tests can be made: for instance, it is found that the addition of a small quantity of calcium to the solution into which the axoplasm is extruded will cause rapid solution of the jelly. Electron microscopy of the axoplasm has revealed elongated filaments showing fine striations.

A major problem which has to be faced by anyone who embarks on research with the giant squid fibre is that of obtaining living squid. With all the resources of the laboratory at Plymouth, and although squid are plentiful in the sea at most seasons of the year, the conditions are such that a specimen which is capable of living for more than $\mathrm{I} 2 \mathrm{hr}$. in the laboratory tanks is a rarity. The cuttlefish is a hardier animal and will live for a long period in captivity. Unfortunately, its nerve fibres are not so large as those of the squid, being only about $\mathrm{I} 50 \mu$ in diameter, and no one has attempted to insert an internal electrode through the cut end of such a fibre. However, they are large enough to possess one advantage over the crustacean fibres because the volume of a single fibre, perhaps $4 \mathrm{~cm}$. long, is just great enough to allow quantitative analysis of its constituents, and an attempt has recently been made to measure the amount of sodium which enters the fibre as the result of activity. Radioactive tracers can be used to follow movements of ions through the membrane, both at rest and when it is stimulated: it is true that this is possible also with the smaller crustacean fibres, but the improvement in accuracy which goes with the use of a larger fibre makes the cuttlefish especially valuable for such purposes.

\section{CONCLUSION}

It should be clear why the nerves of marine animals are much sought after. There is no reason to suppose that, in essential details, the changes in the membrane of the non-myelinated fibre which occur in activity do not also take place at the nodes of Ranvier in a myelinated nerve. The saltatory progression of the impulse in the myelinated nerve is due to a mechanism which should probably be considered as an added, and not very mysterious, refinement. The electrical excitation of one node by another is thought to involve essentially the same process as is found in non-myelinated nerve, by which one part of the membrane is excited by an adjacent one. The vital 'explosive' change at the membrane forms the subject of the most intriguing current research on peripheral nerve, and as it may be predicted that this change, if it is ultimately sufficiently understood, will be found to be fundamentally the same in both types of nerve, one may conclude that much has been learned about vertebrate nerves by studying those of invertebrates. 